\title{
Zur Struktur und Entstehung schwarzer Wismutaufdampfschichten
}

\author{
Von Lothar Fritsche, Franz Wolf und Alfred Schaber \\ Aus dem Institut für Theoretische Physik der Technischen Hochschule Karlsruhe \\ (Z. Naturforschg. 16 a, 31-36 [1961] ; eingegangen am 17. Oktober 1960)
}

Herrn Prof. Dr. W. Hanle zum 60. Geburtstag gewidmet

\begin{abstract}
An schwarzen und spiegelnden Bi-Niederschlägen, die unter stark variierten Bedingungen in verdünnter Luft auf gekühlten Kunststoff-Folien aufgedampft waren, wurden mittels RöNTGEN-Kleinwinkelstreuung Teilchengrößen gemessen. Kleinste Bi-Mengen aus dem Gasraum zwischen Verdampfer und Schichtträger, die sich qualitativ durch TyndalL-Streuung nachweisen ließen, konnten ferner herausgefangen und elektronenmikroskopisch genauer studiert werden. Derartige Untersuchungen führten $\mathrm{zu}$ dem Ergebnis, daß schwarze Schichten durch Niederschlag eines bereits im Raum kondensierten Bi-Nebels auf dem Schichtträger entstehen. Maßgebend für den Vorgang ist nicht nur der Druck der Restgasatmosphäre, sondern außerdem die Geschwindigkeit, die Temperaturverteilung und der Metalldampfdruck innerhalb der Konvektionsströmung zwischen Ofen und Schichtträger. Man kann also bei gleichem Restgasdruck sowohl schwarze als auch metallische sowie Ubergangsschichten erhalten. Erfahrungen anderer Autoren lassen sich diesen Vorstellungen weitgehend einordnen.
\end{abstract}

$\mathrm{Da}$ beim Verdampfen vieler Metalle in Luft oder anderen Gasen bei Drucken von etwa 0,5 Torr schwarze Niederschläge entstehen, ist lange bekannt, z. B. Anm. ${ }^{1-4}$. In restlicher Luft erzeugte Wismutaufdampfschichten dienen technisch als beste Schwärzung für Bolometer, Thermoelemente u. ä. Über das Wesen und den Entstehungsmechanismus solcher Schichten hatte man lange nur unklare Vorstellungen. Das stumpf schwarze Aussehen feinst verteilter Metalle, wie es etwa von Platinmohr bekannt ist, legt nahe, daß auch die Aufdampfschichten eine sehr lockere Struktur aus kleinsten Bauelementen besitzen. Tatsächlich konnten ihre außerordentlich große effektive Oberfläche und gelegentlicher Einschluß großer Gasmengen unmittelbar nachgewiesen werden ${ }^{5}$. Durch geringe mechanische Beanspruchung wie Reiben, Zusammenkratzen u. ä. ist es möglich, den schwarzen Belag in einen silbrig glänzenden überzuführen ${ }^{6}$. Für die lockere Struktur spricht auch, daß z. B. bei Wismut ${ }^{7}$ die elektrische Leitfähigkeit der schwarzen Schicht um viele Zehnerpotenzen kleiner gefunden wird als die der bei gutem Vakuum hergestellten spiegelnd blanken, daß ferner die charakteristische Widerstandsbeeinflussung durch Magnetfelder im schwarzen Zustand ganz verschwindet. Andererseits beweisen Debye-

1 A. H. Pfund, Phys. Rev. 35, 1434 [1930]; Rev. Sci. Instrum. 1, 397 [1930] ; J. Opt. Soc. Amer. 23, 375 [1933].

2 H. C. Burger u. P. H. van Cittert, Z. Phys. 66, 210 [1930].

3 F. Kerkhof, Ann. Phys. 31, 315 [1938].

4 S. Mamiya, Science of Light (Japan), Vol. 1, No. 1, Jan. 1951.

5 Y. TANaka u. M. SakaI, Science of Light (Japan), Vol. 1, No. $1 / 2$.
SCHERRER-Aufnahmen mit Röntgen-Strahlen ${ }^{4}$ und Elektronen, daß die schwarzen Schichten nicht amorph, sondern wie die spiegelnd blanken deutlich aus Kristalliten aufgebaut sind. Es wäre danach denkbar, daß auf dem Schichtträger und an ersten Metallkeimen adsorbiertes Gas bei der Entstehung schwarzer Beläge die Oberflächenwanderung kondensierender Bi-Atome behindert, wodurch viele kleine regellos gelagerte Kristallite mit Lücken entstehen, aber der Aufbau eines größeren zusammenhängenden Kristallgitters wie bei blanken Schichten unterbunden wird.

Hiernach müßte Verdampfung in einer Edelgasatmosphäre statt im Sauerstoff der Luft wegen der fehlenden Chemisorption an den entstehenden Keimen wenn nicht überhaupt blanke, so doch wenigstens mangelhaft geschwärzte Schichten erzeugen. Unsere entsprechenden Versuche in Argon lieferten aber keinerlei Unterschied gegen die früheren $\mathrm{Er}$ gebnisse in Luft. Zudem gibt es von verschiedenen Seiten ${ }^{8,9}$ Hinweise dafür, daß adsorbierte Sauerstoffschichten die Beweglichkeit kondensierter Metallatome nicht hemmen, sondern vergrößern, so daß das Wachstum regelmäßiger Kristalle noch gefördert wird. Die obige Entstehungshypothese für schwarze Schichten trifft also nicht zu.

${ }^{6}$ W. ORT, Diplomarbeit, Karlsruhe 1957. Schwarze Wismutaufdampfschichten.

7 H. WeIss, Diplomarbeit, Karlsruhe 1954. Widerstandsverhalten dünner Wismutschichten im Magnetfeld.

8 H. König u. G. Hellwig, Z. Phys. 129, 491 [1951].

9 K. Hanssen, Z. Phys. 150, 527 [1958]. 


\section{Entstehungsmechanismus schwarzer Schichten}

Aufschlußreich ist die Erfahrung ${ }^{6,7}$, daß auf verschiedenen Auffangeplättchen, die bei einigen Zehntel Torr gleichzeitig einer einzigen Wismutdampfquelle unter verschiedenen Richtungen und in verschiedenen Abständen gegenübergestellt wurden, bei demselben Verdampfungsprozeß sowohl schwarze als auch metallisch blanke Schichten und Übergänge zwischen beiden entstanden. Auf ausgedehnten Auffängern können sich so die wunderlichsten Muster zwischen tiefschwarz und metallisch blank ausbilden. Hier zeigt sich, daß das vom Ofen verdampfte Metall weder als Atomstrahl noch durch Diffusion im Gas in geometrisch definierter Weise zum Auffänger gelangt. Vielmehr bildet sich in der Restatmosphäre des Rezipienten unter Einfluß der großen Temperaturgegensätze ein verwickelter kräftiger Strömungsvorgang aus, und dieser führt, wie weiter unten noch genauer belegt wird, das verdampfte Wismut zum Auffänger. Man kann diese Konvektionsströme bei geeigneter Beleuchtung durch Tyndall-Streuung unmittelbar sichtbar machen. In Abb. ${ }^{*}$ dient als Verdampfungsofen eine elektrisch geheizte, von ihren Zuleitungsdrähten getragene Molybdänblechrinne von etwa $3,5 \mathrm{~cm}$ Länge, eine Auffangplatte wurde nicht angebracht. Die Glasglocke des Rezipienten ist senkrecht zur Blickrichtung mit Licht eines Kohlebogens durchleuchtet. Zweifellos entsteht die Streulichtmarkierung durch Anwesenheit des Metalls, ohne Nachlieferung von Metall bleibt sie völlig aus. Man sieht, das verdampfte Metall wird durch die thermische Luftströmung in ganz unregelmäßigen Schwaden vom Ofen fortgetragen. Für kürzere Zeit bleibt die durch Zufälligkeiten der Anordnung bedingte Verteilung der Strömung über den Raum stationär. Aber man sieht im Gefolge des fortschreitenden Verdampfungsprozesses (Temperaturschwankungen durch Abdampfen, Kondensation des $\mathrm{Bi}$ an anderen Stellen u. a.) auch zeitliche Veränderungen. Eine zeitlich wirklich konstante Strömung mit genau vorausbestimmbarer Geometrie läßt sich praktisch niemals verwirklichen, die Reproduzierbarkeit aller Bedampfungsversuche bei größeren Drucken ist daher beschränkt.

* Abb. 1 und 3-8 auf Tafel S. 32 a.

10 H. Levinstein, J. Appl. Phys. 20, 306 [1949].

11 L. Harris u. J. K. Beasley, J. Opt. Soc. Amer. 42, 134 [1952].
Das TyNDALL-Leuchten ist aber in einer Hinsicht fest mit den Versuchsbedingungen verknüpft: Richtet man diese so ein, daß metallisch blanke Niederschläge entstehen müssen (Einzelheiten weiter unten), so bleibt die Lichtstreuung vollständig aus. Erst wenn man die Aufdampfbedingungen wieder so verändert, daß zunächst stumpfe, matte „Übergangsschichten " und schließlich ganz schwarze Niederschläge entstehen, so taucht anfänglich ein schwaches, dann ein immer stärkeres Leuchten innerhalb der thermischen Strömung auf. Die Lichtstreuung und das Entstehen von Schwarz bei den Schichten gehen streng einander parallel.

Offensichtlich kommen die schwarzen Schichten dadurch zustande, daß bereits im Raum gebildete kompakte Teilchen sich auf dem Schichtträger nach Zufall niederschlagen. Auch von anderer Seite wird diese Auffassung vertreten ${ }^{10-13}$. Bei extremen Versuchsbedingungen kann man unmittelbar mit dem Auge kleine schwarze Bi-Flöckchen beobachten, die frei aus dem strömungsdurchsetzten Raum herausfallen.

Zweifellos ist es das Restgas, das bei größeren Drucken um 0,5 Torr die verdampfenden Metallatome am Fortfliegen hindert, so daß diese zunächst in dichten Wolken zusammenbleiben und so von der Konvektionsströmung erfaßt werden. (Wir denken fernerhin im Anschluß an unsere Versuche immer an Wismut.) Man kann das gaskinetische Verhalten der Bi-Dampfatome hier wohl angemessen erfassen durch ihr mittleres Verschiebungsquadrat $\overline{x^{2}}$ in Luft. Mit der Annahme von Zimmertemperatur für das Restgas und zugehörigem Wert der Viskosität, ferner mit dem Bi-Atomradius ${ }^{14} a=0,92 \AA$ und dem Schätzwert von 1 sec für die Zeit, in der nach den Tyndall-Beobachtungen ein Volumelement der Wismutwolke durch die Strömung vom Ofen bis zum Auffänger getragen wird, ergibt sich

$$
\sqrt{\overline{x^{2}}}=0,84 \mathrm{~mm} \text {. }
$$

Es ist hiernach überhaupt nicht möglich, daß die Bi-Wolke auf dem Weg zum Auffänger merklich auseinanderdiffundiert. Die vom Ofen gelieferte, meist sehr große Dampfschicht bleibt erhalten. Man hat von Anfang an bzw. mindestens mit abnehmen-

12 S. Hirota, Y. Mizushuma u. H. Noake, J. Appl. Phys., Japan 25, 294 [1956].

13 Herrn Dr. E. T Taub, Stuttgart, danken wir hierdurch für seinen Hinweis auf eigene derartige Laborerfahrungen.

14 Landolt-Börnstein, Physikal.-chem. Tabellen. 
L. Fritsche, F. Wolf und A. Schaber, Zur Struktur und Entstehung schwarzer Wismutaufdampfschichten (S. 31).

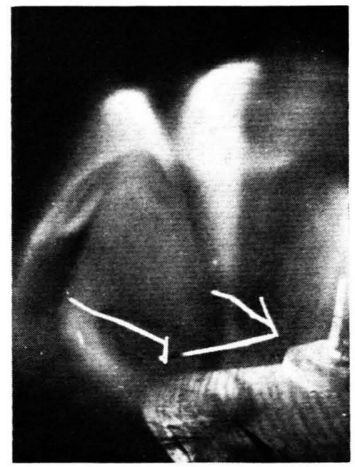

Abb. 1. Konvektionsströmung mit $\mathrm{Bi}$ Nebel im Trndall-Licht.

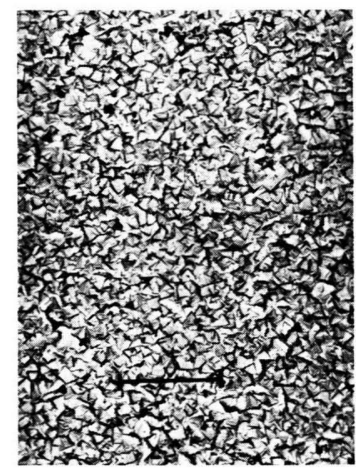

Abb. 3. Elektronenmikroskopisches Bild einer spiegelnden Schicht. $|-| 1 \mu$.

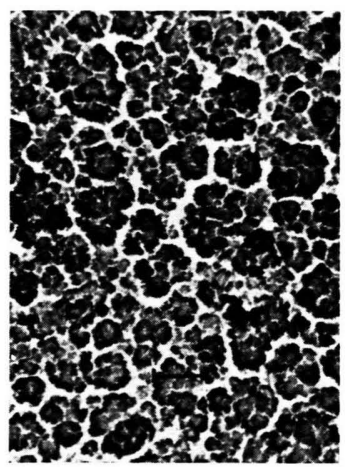

Abb. 4. Elektronenmikroskopisches Bild einer schwarzen Schicht. $|-| 1 \mu$.

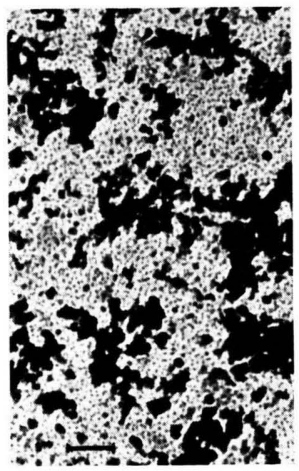

. Bi-Teilchen aus dem Abb. 6. Bi-Teilchen aus dem Abb. 7. Bi-Teilchen aus dem Abb. 8, Bi-Teilchen aus dem Raum, Bedingungen für me- Raum, Bedingungen für Raum, Bedingungen für Raum, Bedingungen für tallische Schicht. - $1 \mu$. Übergangsschicht, größerer schwarze Schicht, großer schwarze Schicht, großer
Dampfdruck.
Abstand,

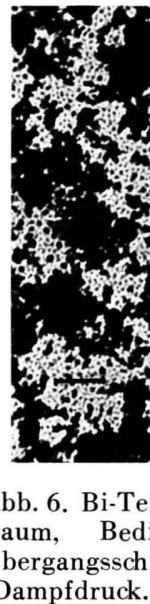

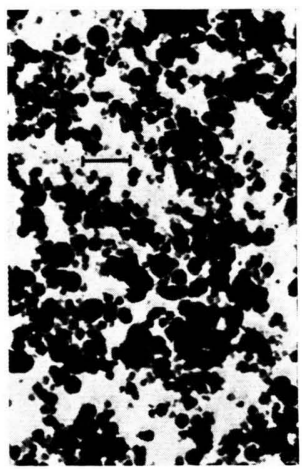

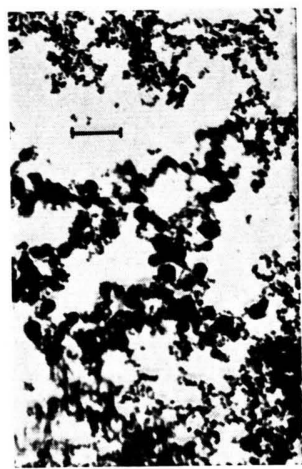

\begin{tabular}{|l|c|c|c|c|}
\hline Abb. Nr. & 5 & 6 & 7 & 8 \\
Druck $p$ & 0,1 & 0,15 & 0,15 & 0,6 \\
Ofenstrom $I$ & 10 & 12,5 & 12,5 & Torr \\
Abstand $s$ & 3,5 & 5 & 15 & 5 \\
$\begin{array}{c}\text { Aussehen fertiger } \\
\text { Schicht }\end{array}$ & metallisch & Übergang & schwarz & schwarz \\
$\begin{array}{l}\text { Teilchengrößen } \\
\text { kleine } \\
\text { größere runde }\end{array}$ & $20-70 \AA$ & $20-70 \AA$ & fast ganz verschwunden & fast ganz verschwunden \\
Haufwerk & $100-200 \AA$ & $100-200 \AA$ & $100-400 \AA$ & $100-300 \AA$ \\
\hline
\end{tabular}

Tab. 2. Elektronenmikroskopische Aufnahmen der Schwebeteilchen. 

der Temperatur des Dampfes auf dem Weg zum Auffänger Sättigung und Übersättigung, so daß Kondensation eintreten kann, wenn Kerne dafür zur Verfügung stehen. In einfacheren Apparaturen sind solche schon in allerlei größeren Fremdmolekülen und Staub, in hochgereinigten immer noch in Ionen und den in kleinen Mengen auftretenden $\mathrm{Bi}_{8}$-Molekeln vorhanden.

Daß der Bi-Dampf auf dem Weg zur Schicht rasch abgekühlt wird und bei genügender Dichte kondensiert, ergibt sich aus den zahlreichen Zusammenstößen seiner Atome mit den Molekeln der Luft, die sich annähernd auf Zimmertemperatur befindet. Der Dampfdruck des Bi (s. Anm. ${ }^{15}$ ) bei der Ofentemperatur von $850{ }^{\circ} \mathrm{C}$ beträgt ungefähr 0,3 Torr. Dies bedeutet eine Teilchendichte des Dampfes von $2,6 \cdot 10^{15} \mathrm{~cm}^{-3}$. Aus ihr und dem obigen Atomradius folgt die mittlere freie Weglänge

$$
\text { für } \mathrm{Bi} \text { in Bi-Dampf } \quad \lambda=2,6 \mathrm{~mm} \text {. }
$$

Dagegen wird mit Hilfe der Teilchendichte der restlichen Luft (0,5 Torr), dem gaskinetischen Wirkungsradius der Luft von 1,84 $\AA$ und demselben Bi-Atomradius wie vorher die mittlere freie Weglänge

$$
\text { für Bi in Luft } \quad \lambda=0,17 \mathrm{~mm} \text {. }
$$

Stöße der Wismutatome mit Luftmolekülen sind also mehr als zehnmal so häufig wie diejenigen der Bi-Atome untereinander. Der Dampf wird sehr rasch abgekühlt und bei genügender Dichte kondensiert.

Die Kondensation kompakter Schwebeteilchen aus dem Dampf hat zur Voraussetzung einmal genügend großen Gasdruck, andererseits aber auch hinreichende Dampfdichte, die durch die Ofentemperatur reguliert wird. Beide Bedingungen müssen erfüllt sein, damit schwarze Niederschläge erhalten werden. Dies konnte ORT experimentell bestätigen ${ }^{6}$. Abb. 2 zeigt seine Ergebnisse. Statt des Schwärzungsgrades ist hier als Ordinate die von den erhaltenen Schichten im weißen Licht reflektierte Intensität aufgetragen, bezogen auf den Wert des kompakten Bi-Spiegels. Als Abszisse dient an Stelle der vom Ofen gelieferten Dampfdichte die leichter meßbare „Auftreffrate", d. h. etwa die auf der Schicht ankommende Atomstromdichte. Man sieht, daß man über einen weiten Bereich verschiedener Gasdrucke von schwarzen Schichten (unten) bis zu völlig spiegeln-

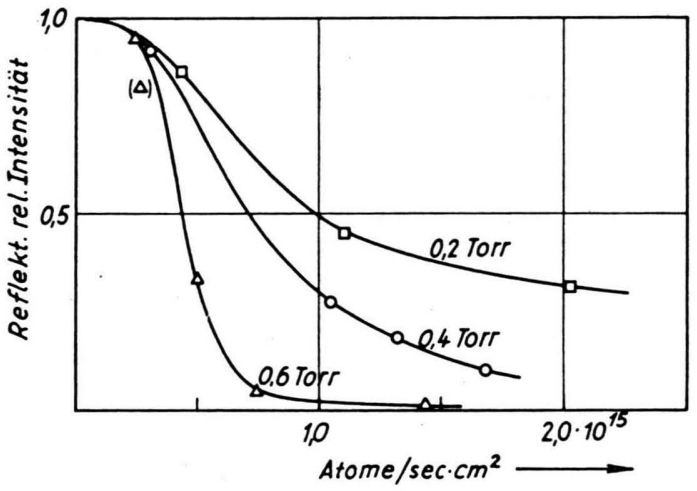

Abb. 2. Reflektierte Lichtintensität über der Auftreffrate bei verschiedenen Drucken.

den (oben) übergehen kann, wenn man nur die Auftreffrate und damit die vom Ofen stammende Dampfdichte von großen Werten (rechts) bis zu hinreichend kleinen (links) herabsetzt.

Außer Gasdruck und Metalldampfdichte ist die Transportgeschwindigkeit des Dampfes bzw. seines Kondensats für das Bedampfungsergebnis maßgebend. Sie ist praktisch mit der Geschwindigkeit der Konvektionsströmung identisch, die von kaum erfaßbaren Parametern abhängt. Die Deutung der Bedampfungsergebnisse wird hierdurch vielfach erschwert.

Bei geringem Abstand zwischen Ofen und Auffänger kann infolge Zustrahlung die Temperaturdifferenz zwischen beiden so gering sein, daß Sättigung des Bi-Dampfes auf dem Weg zum Schichtträger überhaupt nicht oder kaum erreicht wird, die Kondensation also im wesentlichen unterbleibt. Damit entsteht aus den herangetragenen Dampfatomen eine metallisch spiegelnde Schicht, während sich gleichzeitig auf einem weiter entfernt angebrachten Auffänger eine schwarze ausbilden kann.

\section{Teilchengrößen durch Röntgen-Kleinwinkel- streuung}

Abb. 3 und 4 zeigen durch Kohleabdruck erhaltene elektronenmikroskopische Aufnahmen von $\mathrm{ORT}^{6}$ von der Oberfläche eines spiegelnden und eines schwarzen Bi-Niederschlags. Der spiegelnde besteht aus Kriställchen, von denen etwa vier bis fünf aneinandergelegt die Länge einer Welle des sichtbaren Lich. tes ausmachen. Die elektronenmikroskopische Teilchengröße spiegelnder Schichten kann allerdings je

15 M. v. Ardenne, Tabellen d. Elektronen-, Ionenphysik und Ubermikroskopie, 1956. 
nach Versuchsbedingungen in sehr weiten Grenzen schwanken ${ }^{16}$. - Im folgenden sollen in erster Linie die Eigenschaften stumpfer und schwarzer Niederschläge interessieren. Die Abb. 4 einer schwarzen Schicht zeigt viel grobere Struktur als die spiegelnde, mehrere Lichtwellenlängen fallen auf den mittleren Periodizitätsabstand. Gleichzeitige stereoskopische Aufnahme ergab eine große Tiefenerstreckung. Es sieht aus, als ob man von oben herab Baumkronen eines Laubwalds betrachtete.

Nehmen wir Ergebnisse von OrT über die Linienbreite von Debye-Scherrer-Bildern mit eigenen zusammen, so bewegen sich die Größen der aufbauenden Einzelkristallite bei schwarzen Schichten (Herstellungsdruck $0,6-0,8$ Torr) zwischen etwa 50 und $95 \AA$, sind also wesentlich kleiner als die „Baumkronen".

Ein weiteres Verfahren, das speziell über die Größe individueller, räumlich getrennter Teilchen Aufschluß gibt, ist die Methode der Röntgen-Kleinwinkelstreuung ${ }^{17-19}$. Um bei der Herstellung der hierbei benutzten Aufdampfschichten definierte Temperaturverhältnisse zu haben, wurden sie stets in etwa $4 \mathrm{~cm}$ Abstand auf Hostaphanfolien niedergeschlagen, die von der Rückseite mit fließendem Leitungswasser gekühlt waren. Ihre Analyse durch RöntGen-Kleinwinkelstreuung erfolgte im wesentlichen nach der Methode von Shull und Roess ${ }^{20}$ mittels Kupfer-K $\mathrm{K}^{-}$-Strahlung, jedoch mit einer Reihe von Sicherungen und Verfeinerungen, unter denen vor allem die Verwendung einer KRATKY-Blende ${ }^{21,22}$ hervorzuheben ist. Es gelang, das Auflösungsvermögen so weit zu steigern, daß noch Teilchen mit dem Durchmesser $740 \AA$ als oberer Grenze meßbar waren ${ }^{23}$. Tab. 1 zeigt einige Ergebnisse. Die Aufdampfbedingungen sind jeweils festgelegt durch die Auffängertemperatur (konstant $15^{\circ} \mathrm{C}$ ), durch den Gasdruck $p$ und die Dichte des Bi-Dampfes, die hier indirekt durch die Ofenstromstärke $I$ erfaßt wird. Oberhalb von $10 \mathrm{Amp}$ steigt sie steil an. Das bei verschiedenen Bedingungen erhaltene Aussehen der Schichten bestätigt die oben entwickelten Vorstellungen über ihr Zustandekommen.

16 M. Auwärter, Ergebnisse der Hochvakuumtechnik und der Physik dünner Schichten, Wissenschaftl. Verlagsgesellsch. m.b.H., Stuttgart 1957. L. Holland, Vacuum deposition of thin films, Champman \& Hall Ltd., London 1958.

17 R. Hosemann, Erg. exakt. Naturw. 24, 142 [1951].

18 O. Kratky, Z. Elektrochem. 60, 245 [1956].

10 W. Beeman, P. Kaesberg u. Mitarb., Handbuch der Physik, Bd. 32, S. 321 [1957].

\begin{tabular}{|c|l|l|c|}
\hline $\begin{array}{c}p \\
(\text { Torr })\end{array}$ & $\begin{array}{c}I \\
(\mathrm{~A})\end{array}$ & \multicolumn{1}{|c|}{ Aussehen der Schicht } & $\begin{array}{c}\text { Durch- } \\
\text { messer } \\
(\AA)\end{array}$ \\
\hline $10^{-3}$ & 10 & hell, metallisch glänzend & 270 \\
0,12 & 10 & Übergang von metallisch zu & 280 \\
0,15 & 12,5 & $\begin{array}{c}\text { schwarz, glänzend } \\
\end{array}$ & \\
0,3 & 11 & braunschwarz, glänzend & 220 \\
0,6 & 12 & samtartiz schwarz & 260 \\
1,0 & 12 & samtartig braunschwarz & 260 \\
\hline
\end{tabular}

Tab. 1. Teilchendurchmesser bei Schichten auf Hostaphanfolien.

$\mathrm{Da}$ für die metallisch glänzenden Schichten hier andere Teilchengrößen herauskommen als oben bei der elekrtonenmikroskopischen Abb. 3, zeigt nur, $\mathrm{da} ß$ verschiedene Erzeugungsbedingungen vorgelegen haben. Sehen wir von den metallisch glänzenden Niederschlägen $a b$, so bestehen sowohl die Übergangsschichten wie die schwarzen Schichten nach diesen Messungen aus Teilchen ungefähr gleicher mittlerer Größe von etwa $250 \AA$. Die von der Kleinwinkelstreuung erfaßten Partikel erweisen sich damit als wesentlich kleiner als die „Baumkronen“ von Abb. 4. Diese sind daher bestimmt Haufwerke aus kleineren Bausteinen. Wie weit die Bausteine ihrerseits identisch sind mit den im Raume kondensierten Bi-Teilchen, wurde an weiteren elektronenmikroskopischen Aufnahmen untersucht.

\section{Elektronenmikroskopische Studien}

Da es aussichtslos erscheint, das Wismut auf seinem Weg zum Schichtträger frei im Raum schwebend zu erfassen, haben wir versucht, es bei verschiedensten Aufdampfbedingungen aus dem Flug möglichst unverfälscht auf elektronenmikroskopischen Objektträgern aufzufangen. Um dabei Veränderungen durch zu starke Erhitzung vor dem Verdampfungsofen bzw. bei der elektronenmikroskopischen Durchstrahlung zu vermeiden, wurden die Expositionszeiten so kurz wie möglich gehalten (wenige Zehntelsekunden bei der Bedampfung). Die Aufnahmen, für die die Abb. 5, 6, 7, 8 Beispiele geben, zeigen dem-

20 C. Shull u. L. Roess, J. Appl. Phys. 18, 295, 308 [1947].

21 O. Kratky, Z. Elektrochem. 58, 49 [1954].

22 O. Kratky u. Z. Skala, Z. Elektrochem. 62, 66, 73 [1958].

23 Wegen aller Einzelheiten siehe A. Schaber, Diplomarbeit, Karlsruhe 1960. Untersuchungen über die Entstehung und die Struktur schwarzer und metallischer Wismutaufdampfschichten. 
nach nur eine sehr lückenreiche Anfangsbedeckung der Bildebene durch einzelne Teilchen, während eine fertige Aufdampfschicht bei dieser Wiedergabe völlig undurchsichtig erscheinen müßte.

In Tab. 2 sind die zu den Abb. 5 bis 8 gehörenden Versuchsbedingungen nebst den Ergebnissen zusammengestellt. Die Auffängertemperatur ist hier nicht genau angebbar, sie dürfte größer als bei den fertigen Schichten auf gekühltem Hostaphan gewesen sein. Zusätzlich wurde diesmal auch der Abstand des Objektträgers vom Verdampfungsofen variiert $(s)$. Vor den erhaltenen Teilchengrößen steht noch das Aussehen einer fertig aufgedampften Schicht, das man unter den betreffenden Bedingungen zu erwarten hätte.

Auf den Bildern fällt zunächst auf, daß bei jedem der gewählten Drucke in kürzester Zeit kompakte Metallgebilde nachgewiesen werden, bei denen die kugelige Form vorherrscht, während man im Hochvakuum unter einem Metallatomstrahl nach einiger Zeit Andeutungen von Kristalliten finden würde. Offenbar schlägt sich das Metall hier wirklich bereits in Form größerer kompakter Teilchen aus dem Raum nieder. Die Kugelform spricht dafür, daß flüssige Tropfen vorgelegen haben. Freilich läßt sich nicht sagen, ob diese im Raum oder beim Auftreffen auf den Schichtträger erstarrt sind. Es könnte sogar sein, daß die Tropfenform erst vom Wiederaufschmelzen bereits verfestigter Teilchen auf dem Objektträger durch zu große Erwärmung herrührt. Doch spricht die völlige Unabhängigkeit der Form von den stark variierten Versuchsbedingungen der Tab. 2 nicht dafür. Weiter ist die Frage, wie die sehr verschiedenen Radien, wie ferner die häufig auftretenden größeren Anhäufungen zu verstehen sind. Man kann auf den Aufnahmen ja deutlich unterscheiden zwischen sehr kleinen Teilchen, zwischen größeren von ausgesprochener Kugelgestalt und schließlich grobem Haufwerk, das hauptsächlich aus Kugeln zusammengesetzt zu sein scheint. Die oben mittels RöNTGEN-Kleinwinkelstreuung gewonnenen Durchmesser sind offenbar Mittelwerte über diese Größenverteilung. Auf (nicht wiedergegebenen) stereoskopischen Aufnahmen sieht man vielfach ganze Ketten aneinandergelagerter Kugeln wechselnder Größe, die oft aus der Trägerebene weit in den Raum hinausragen. In Abb. 8 deuten sich solche Gebilde durch ihre Unschärfe an, da sie sich unter Erwärmung durch Elektronenbestrahlung (auf dem Leuchtschirm unmittelbar sichtbar) hin- und her- bewegen. Das grobe Haufwerk der Bilder dürfte in ähnlicher Weise dreidimensional aus Kugeln zusammengesetzt sein.

Die Größe der aus Bi-Dampf kondensierten Tropfen hängt sowohl von dem angebotenen Dampfdruck als auch von der zum Wachsen verfügbaren Zeit ab. Auch die Art der Kondensationskerne, etwa ob Moleküle oder mikroskopische Stäubchen, spielt eine wesentliche Rolle. So können sehr wohl Tröpfchen verschiedener Größe nebeneinander wachsen. In Gebieten der Verarmung an Bi-Dampf mag sogar ein Wiederverdampfen der kleineren Tröpfchen mit überhöhter Oberflächenspannung einsetzen, so daß auf längerem Weg die großen die kleinen Tröpfchen aufzehren. Dies ist vielleicht die Erklärung dafür, daß die in der Nähe des Ofens beobachteten kleinsten Tröpfchen (Abb. 5, 6) in größerer Entfernung und bei größerem Gasdruck (Behinderung der Dampfdiffusion) verschwinden (Abb, 7, 8) . - Das Haufwerk kann durch Aufeinandertreffen zahlreicher Tropfen auf dem Träger, auf gleiche Weise aber auch schon im Raum entstanden sein, da verschiedene Tropfengrößen mit verschiedenen Geschwindigkeiten von der Konvektionsströmung mitgenommen werden. Es dürfte wohl solches Haufwerk sein, das sich auf der Oberflächenaufnahme von Abb. 4 als "Baumkronen“ abzeichnet. Dabei werden die sie aufbauenden Einzeltropfen im festen Zustand selbst noch meist polykristallin aus den durch DebyeScherrer-Aufnahmen nachgewiesenen Einzelkristalliten zusammengesetzt sein.

Die Abhängigkeit des Tropfenwachstums vom gebotenen Dampfdruck wird deutlich aus den Abb. 5 und 6, bei denen Gasdruck und Entfernung wenigstens annähernd übereinstimmen. Auf Abb. 6, bei der größerer Heizstrom viel größere Dampfmengen geliefert hat, findet sich viel mehr und größeres Haufwerk als auf Abb. 5. Für die Abhängigkeit des Wachstums von der Zeit liefern die Abb. 6 und 7 ein Beispiel. Bei gleichem Gasdruck und gleicher, übrigens großer Dampfdichte sind in größerer Entfernung $s$ vor allem die mittleren kugeligen Teilchen, wahrscheinlich auf Kosten der verschwundenen kleinsten, außerordentlich angewachsen.

Schwierig zu beantworten ist die Frage, warum man im Fall der Abb. 5 eine metallische Schicht erwarten muß, obwohl auch hier bereits kondensierte Teilchen aus dem Raum einfallen. - Sicher hat man daneben, in der kurzen Expositionszeit nur nicht deutlich sichtbar, auch einen erheblichen Strom ato- 
maren Wismuts, das sich auf dem Träger zu Kristalliten aufbauen und eine dichte Metallstruktur anstreben wird.

Eine sauber kristalline Struktur wie in Abb. 3 ist vielleicht gar nicht erforderlich. Damit eine Aufdampfschicht metallisch spiegelt, scheint sie vor allem eine vergrößerte elektrische Leitfähigkeit $\sigma \mathrm{zu}$ benötigen. Dies geht aus Untersuchungen von $\mathrm{HAR}_{\mathrm{AR}}$ RIS und BEAsLEy ${ }^{11}$ hervor, denen es gelang, das Verhalten schwarzer Niederschläge wenigstens im nahen Ultrarot mittels der klassischen Metalloptik quantitativ wiederzugeben. In dem bekannten Ansatz

$$
R=\frac{\left(n_{0}-1\right)^{2}+k^{2}}{\left(n_{0}+1\right)^{2}+k^{2}}
$$

für das Reflexionsvermögen sind $n_{0}$ und $k$ die Bestandteile des komplexen Brechungsexponenten $n=n_{0}-i k$, die mittels

$$
\left.\begin{array}{c}
n_{0} \\
k
\end{array}\right\}=\left\{\frac{\varepsilon_{\mathrm{r}} \mu_{\mathrm{r}}}{2}\left(\sqrt{\left.1+\eta^{2} \pm 1\right)}\right\}^{1 / 2} \text { mit } \eta=\frac{\sigma \lambda}{2 \pi c \varepsilon_{\mathrm{r}} \varepsilon_{0}}\right.
$$

aus den elektrischen Eigenschaften des Spiegels und der Lichtwellenlänge folgen. Es zeigt sich, daß man hier wegen der außerordentlich großen Porosität der schwarzen Schichten mit Erfolg für DK und Permeabilität die Vakuumwerte $\varepsilon_{\mathrm{r}}=\mu_{\mathrm{r}}=1$ verwenden kann. Hiermit wird $\eta$ und mit ihm das Reflexionsvermögen außer durch die Wellenlänge nur durch die Leitfähigkeit maßgeblich bestimmt. Da diese bei den porösen schwarzen Schichten außerordentlich klein ist, erhält man den einfachen Näherungsausdruck $R=\eta^{2} / 16$, der - in qualitativer Übereinstimmung mit der Erfahrung - an der kurzwelligen Grenze des Ultrarot nur etwa $0,01 \%$ liefert.
Wenn nun aber bei der obigen Schicht von Abb. 5 durch eng anschließenden Einbau atomaren Wismuts zwischen den aus dem Raum eingefallenen größeren Blöcken die Leitfähigkeit und damit die Hilfsgröße $\eta$ wesentlich heraufgesetzt wird, so ist damit auch eine entsprechende Steigerung des Reflexionsvermögens wahrscheinlich. Mit $\varepsilon_{\mathrm{r}}=\mu_{\mathrm{r}}=1$ bekommt man jedenfalls nach den obigen Ansätzen

für bzw. $\quad \eta=1, \quad 10, \quad 100$,

$$
R=4,7, \quad 40,2, \quad 75,5 \% \text {. }
$$

Zum Abschluß sei betont, daß sich das Zustandekommen und Verhalten schwarzer Aufdampfschichten, sobald die oben im einzelnen diskutierten Bedingungen erfüllt sind, als weitgehend unabhängig von der Art des benutzten Ofens und vom Reinheitsgrad des Rezipientengases erweist. Danach scheinen früher beobachtete Einflüsse, wie die Mitwirkung verdampften Wolframs bzw. von Sauerstoff ${ }^{24}$, für die Entwicklung der schwarzen Schicht nicht entscheidend zu sein. Wenn schließlich durch Kathodenzerstäubung und durch Explosion von Drähten trotz schlechter Vakua metallische Spiegel entstehen, so dürfte im ersten Fall die geringe Stromdichte, im zweiten der Umstand verantwortlich sein, daß es bei der Explosion gar nicht zu der notwendigen Durchmischung des Metalldampfes mit dem Gas kommt.

Die elektronenmikroskopischen Aufnahmen verdanken wir Herrn Dr. Albert, dem Leiter des Laboratoriums für Elektronenmikroskopie der Technischen Hochschule Karlsruhe. Der D e u t s chen For s chung s ge meins ch af $t$ danken wir für die Gewährung apparativer Hilfsmittel.

24 P. G. Wilkinson, J. Appl. Phys. 22, 226 [1951]. 